\title{
Editorial
}

\section{A paradigm shift for erythropoietin: no longer a specialized growth factor, but rather an all-purpose tissue-protective agent}

\author{
R Savino $^{1, *}$ and G Ciliberto ${ }^{2}$ \\ 1 Dipartimento di Medicina Sperimentale e Clinica, Università di Catanzaro \\ 'Magna Graecia', via Tommaso Campanella 115, 88100 Catanzaro, Italy \\ 2 I.R.B.M. P. Angeletti, Via Pontina km 30,600, 00040 Pomezia, Italy \\ * Corresponding author: R Savino; E-mail: savino@unicz.it
}

Cell Death and Differentiation (2004) 11, S2-S4. doi:10.1038/sj.cdd.4401469

Life science investigators must be prepared to continuously change their minds about biological processes and functions during their career, in order to keep pace with evolving concepts and breakthrough discoveries. Apoptosis is one of the areas of biology in which this mental flexibility is most needed, as there has been considerable progress over the past few years. The review presented in this issue of CDD by Ghezzi and Brines ${ }^{1}$ provides an example of this, by showing how an old cytokine - previously known, and commercially exploited, for its unique role in the proliferation and differentiation of the haematopoietic system - has revealed an unsuspected function as an antiapoptotic factor throughout the body, particularly in the survival of neurons.

Erythropoietin (EPO) has long been known as the glycoprotein hormone that regulates erythropoiesis in mammals. In the adult, it is secreted mainly by kidney peritubular cells in response to hypoxia. In the early 1990s, it was reported that EPO promoted the proliferation of late erythroid precursors and their terminal differentiation into erythrocytes. ${ }^{2}$ Although incomplete, these discoveries led to the development of recombinant EPO for anaemia in 1989, which has been one of the most successful biological drugs so far. It is currently used to control erythrocyte levels following chronic renal failure or in the supportive care of cancer patients undergoing chemoablative regimens.

The review by Ghezzi and Brines sheds new light on EPO, and points to a previously underestimated antiapoptotic function. Although EPO can effectively support the proliferation of erythroid progenitor cells and induce the entry of dormant erythroid progenitors into the cell cycle, ${ }^{3}$ promotion of survival due to prevention of apoptosis of late erythroid progenitors is now presented as a major mechanism of EPO action. ${ }^{4}$ After explaining the haematopoietic activity of EPO by inhibiting apoptosis, Ghezzi and Brines go on to describe the prominent role recently defined for EPO as a neuroprotective agent, which has elicited a growing interest in the potential therapeutic use of EPO for neuroprotection. An impressive number of recent publications are described that demonstrate the protective effect provided by EPO in various in vivo models of brain injury in mouse, rat, rabbit, gerbil and pig. Remarkably, a pilot clinical trial in stroke patients has confirmed the practical relevance of these results. ${ }^{5}$ Evidence of in vivo antiapoptotic effects was not restricted to erythroid progenitor cells and the brain, as several papers demonstrated protection in the retina, spinal cord, and sciatic and peripheral nerves.

The signalling pathways used by EPO to prevent apoptosis are discussed in depth by Ghezzi and Brines, with particular emphasis on EPO signalling in erythroid and in nervous system cells. The authors also state that the cytokine has antiapoptotic effects on cardiomyocytes in vitro and in vivo, as shown using a rat model of myocardial infarction, where it normalizes haemodynamic functions. Among other tissueprotective activities, EPO has been shown in vitro to antagonize the apoptosis of endothelial cells subjected to ischaemic stressors and to prevent apoptosis of tubular epithelial cells in kidney ischaemic injury with reperfusion. Finally, the skin and the intestine have also been shown to be protected by EPO in rat models.

The emerging picture is therefore quite complex (Figure 1) and points to EPO being a central cytoprotective factor, which plays a role in several body compartments when they are undergoing stress. However, as it was originally discovered, EPO is also triggered by hypoxia. An immediate response of the organism to hypoxia is the activation of the central transcription factor HIF; HIF rapidly orchestrates the induction of a set of homeostatic responses - which include production of EPO and, more importantly, vascular endothelial growth factor (VEGF) - that are responsible for counteracting hypoxia and protecting from tissue damage (reviewed in Bruick ${ }^{6}$ and Giaccia et $a .^{7}$ ). The minute amounts of EPO produced under many of these circumstances would not be sufficient to trigger systemic effects that would lead to a significant increase in erythrocytes, but would act locally in a paracrine fashion to maintain tissue homeostasis. This fail-safe mechanism inactivates itself, as soon as normal local oxygen pressure is restored through tissue repair and increased blood flow.

The demonstrated role of EPO as a general tissueprotective cytokine opens up boundless possible therapeutic applications. However, as Ghezzi and Brines point out, after systemic administration, recombinant human EPO encounters cellular barriers on its way to the brain, retina and spinal cord, and the various experimental models have shown that, because of these barriers, the minimum effective dose necessary to obtain a biological effect in the above-mentioned organs is generally higher than that used for treating anaemia. Although acute (i.e. a few doses) administration of recombinant human EPO for the treatment of tissue injury is not likely to be harmful, prolonged dosage would result in increases in erythrocyte mass. This is highly undesirable, as it could 


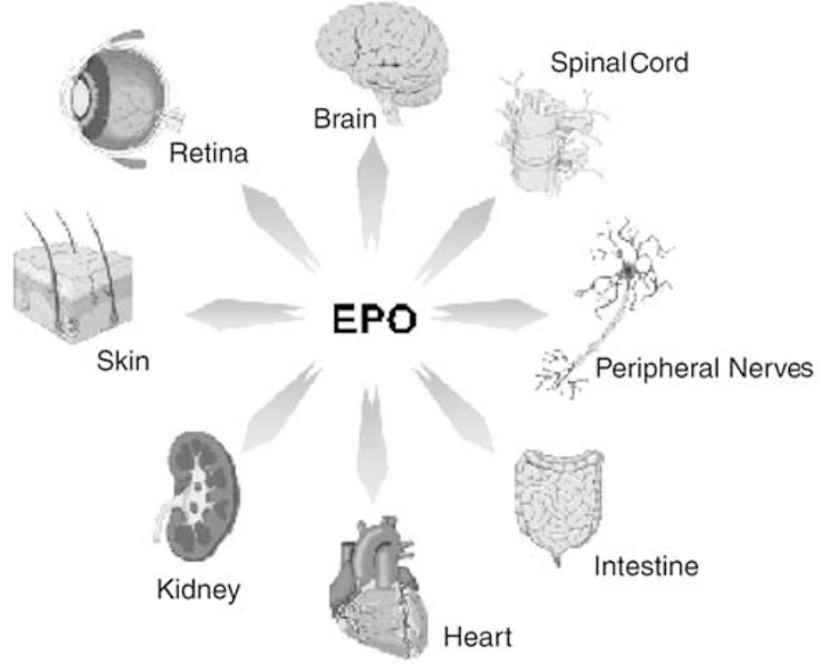

Figure 1 EPO functions on many tissue types. EPO acts as a cytoprotective factor that has a role in several body compartments when they are undergoing stress. These include the brain, spinal cord, peripheral nerves, intestine, heart, kidney, skin and retina

exacerbate tissue injury and cause severe side effects such as increased blood pressure and local thrombosis.

A viable alternative to overcome the undesirable side effects caused by systemically administering high doses of EPO would be to induce high local production of EPO where it is needed. Such a goal can, in theory, be attained using somatic gene transfer, and EPO has been one of the most studied transgenes. Either the EPO gene or cDNA have been delivered in vivo through a variety of gene therapy vectors, including replication-defective first-generation ${ }^{8}$ and helperdependent ${ }^{9,10}$ adenoviral vectors, retroviral vectors, ${ }^{11}$ genetically modified myoblasts, ${ }^{12}$ adeno-associated viral (AAV) vectors $^{13}$ and naked DNA - the transduction efficiency of which can be improved by electroporation ${ }^{14}$ or by a femtosecond infrared titanium sapphire laser beam. ${ }^{15} \mathrm{~A}$ very recent publication demonstrates that a single intravenous injection of naked plasmid DNA encoding EPO provides neuroprotection in hypoxia-ischaemia rats. ${ }^{16}$

It would be interesting to study the neuroprotection afforded by EPO cDNA administration by Herpes Simplex Virus (HSV) vectors. Herpes viruses hold promise as vehicles for the transfer of genes to cells in vivo, because of their ability to persist in a state of latency after primary infections in humans, allowing prolonged expression of the transferred gene. The wild-type virus has a natural tropism for sensory neurons and so the most promising use of these vectors is in gene transfer to neurons. Indeed, HSV vectors have now been successfully tested pre-clinically for the treatment of peripheral nervous system diseases, certain brain diseases, spinal nerve injury and pain (reviewed in Kay et al. ${ }^{17}$ ). Considering their natural tropism, HSV vectors should, in theory, be ideal for local, highlevel expression of EPO, allowing the cytokine to exert its neuroprotective action while minimizing unwanted side effects. Obviously, EPO should still be used with caution, as uncontrolled EPO production can lead to undesirable effects. Significant efforts have been made to achieve stringent transcriptional control of gene-delivered EPO using various vectors, and the results have been recently published by our group (reviewed in Toniatti et al. ${ }^{18}$ ). Over the next few years, one can envisage that stable vectors will be generated, which will permit EPO delivery in well-defined body compartments and tight pharmacological control of its expression.

To conclude, new discoveries about this old cytokine have not only led to a better understanding of its physiological role, but have also opened up new avenues for the treatment of brain disorders. The next few years will require greater insight into whether this role of biological neuroprotection is confined to acute post-ischaemic lesions or could also be extended to chronic neurodegenerative diseases such as Alzheimer's or Parkinson's. Another area of EPO to be further examined is what activity, if any, this cytokine performs on neuronal stem cells. In fact, Ghezzi and Brines state that EPO and the EPO receptor (EPOR) are highly prominent during fetal brain development - the very high expression levels found in many tissues diminish rapidly after birth to the generally low levels found in the adult. Gene knockout experiments have confirmed the importance of this system in development, as the embryos of $\mathrm{EPOR}^{-1-}$ animals have abnormal brain development that is characterized by decreased neuronal progenitor cells, as well as grossly decreased neuronal densities that are associated with massive neuronal apoptosis. Interestingly, a link between neuronal stem cells and haematopoietic conversion has been suggested. ${ }^{19,20}$ Although more recent studies have been unable to confirm this evidence, ${ }^{21}$ neuro-haematopoietic conversion still remains the object of intense debate and controversy. The study of the EPO signalling pathway in these cells may allow us to finally clarify this issue.

\section{Key points}

- Erythropoietin (EPO) is known to be the main hormone responsible for maintaining the erythrocyte mass. It acts on erythropoietic progenitor cells as a differentiation and growth factor, and has recently been shown to have a survival role by acting as an antiapoptotic agent.

- Recent evidence has shown that EPO is able to sustain antiapoptotic responses in several tissues and can be regarded as a general tissue-protective cytokine.

- Among the various antiapoptotic activities, neuroprotection is emerging as one of the most important. EPO has been shown to be effective in several preclinical stroke models and in a recent proof-of-concept clinical study, but may extend to include other chronic CNS diseases.

- Gene transfer of regulated EPO to the brain may be regarded as a viable therapeutic strategy.

1. Ghezzi P and Brines M (2004) Cell Death Differ. 11(S1): S37-S44

2. Krantz SB (1991) Blood 77: 419-434

3. Spivak JL et al. (1991) Blood 77: 1228-1233

4. Silva $\mathrm{M}$ et al. (1996) Blood 88: 1576-1582

5. Ehrenreich $\mathrm{H}$ et al. (2002) Mol. Med. 8: 495-505

6. Bruick RK (2003) Genes Dev. 17: 2614-2623

7. Giaccia A et al. (2003) Nat. Rev. Drug Discov. 2: 803-811

8. Svensson EC et al. (1997) Hum. Gene Ther. 8: 1797-1806

9. Maione $D$ et al. (2000) Hum. Gene Ther. 11: 859-868

10. Maione D et al. (2001) Proc. Natl. Acad. Sci. USA 98: 5986-5991 
11. Yanay $O$ et al. (2003) Hum. Gene Ther. 14: 1587-1593

12. Rinsch $\mathrm{C}$ et al. (1997) Hum. Gene Ther. 8: 1881-1889

13. Ye X et al. (1999) Science 283: 88-91

14. Rizzuto $G$ et al. (1999) Proc. Natl. Acad. Sci. USA 96: 6417-6422

15. Zeira E et al. (2003) Mol. Ther. 8: 342-350

16. Wang $\mathrm{CH}$ et al. (2004) Biochem. Biophys. Res. Commun. 314: 1064-1071
17. Kay MA et al. (2001) Nat. Med. 7: 33-40

18. Toniatti $C$ et al. (2004) Gene and Cell Therapy. Therapeutic Mechanisms and Strategies 2nd edn. Marcel Dekker, Inc.: New York pp. 413-446

19. Bjornson C et al. (1999) Science 283: 534-537

20. Shih $\mathrm{C}$ et al. (2001) Blood 98: 2412-2422

21. Morshead C et al. (2002) Nat. Med. 8: 268-273 